\title{
A Logical Topology Design with Tabu Search in IP over WDM Optical Networks
}

\author{
Galy $\mathrm{LEE}^{1}$, Sugang $\mathrm{XU}^{2}$, and Yoshiaki TANAKA ${ }^{1,3}$ \\ ${ }^{1}$ Global Information and Telecommunication Institute, Waseda University \\ 1-3-10 Nishi-Waseda, Shinjuku-ku, Tokyo, 169-005 l Japan \\ ${ }^{2}$ National Institute of Information and Communications Technology \\ 4-2-1 Nukui-Kitamachi, Koganei, Tokyo, 184-8795 Japan \\ ${ }^{3}$ Advanced Research Institute for Science and Engineering, Waseda University \\ 17 Kikuicho, Shinjuku-ku, Tokyo, 162-0044 Japan \\ E-mail: galylee@fuji.waseda.jp,xsg@nict.go.jp, ytanaka@waseda.jp
}

\begin{abstract}
Given a static traffic condition, the motivation of logical topology design in IP over WDM networks is to optimize the network resource utilization and improve network performance. The objectives of the optimization can be to minimize network congestion or to reduce packet delay in the networks. The logical topology design for IP over WDM networks can be decomposed into several subproblems, some of those subproblems have been proven to be NP-hard problems, and many heuristic algorithms have been proposed to find the near optimal solutions. Our research effort has been put into the investigation of different heuristic algorithms for the logical topology design problem. Using an indirect neighbour discovery method to change the logical topology of the network, several simulated annealing and genetic algorithms for logical topologic design problem in WDM networks have been proposed in our previous researches. In this paper, a tabu search (TS) method to resolve the logical topology design problem is proposed. Our motivation is to find a feasible meta-heuristic algorithm for logical topology design in IP over WDM networks.
\end{abstract}

Index Terms - Meta-heuristic, WDM networks, Tabu search Simulated annealing, Logical topology design

\section{INTRODUCTION}

The wavelength division multiplexing (WDM) technology enables the optical network to provide huge capacity for the network traffic transportation. In the recent years, "All Optical Networks" with WDM has become a hot research area. Given a static traffic condition, the motivation of logical topology design in IP over WDM networks is to optimize the network resource utilization and improve network performance. The objectives of the optimization are to minimize the network congestion or to reduce the packet delay in the network. The logical topology for IP over WDM networks can be decomposed into several subproblems, some of those subproblems are proven to be NP-hard problems, and many heuristic algorithms have been proposed to find the near optimal solutions. Our research effort has been put into the investigation of different heuristic algorithms for the logical topology design problem. Using an indirect neighbour discovery method to change the topology of the network, several simulated annealing (SA) and genetic algorithms (GA) for logical topologic design problem in WDM networks have been proposed in our previous researches. In this paper, our research motivation is to find a feasible meta-heuristic algorithm for logical topology design in IP over WDM networks.

A tabu search (TS) method to resolve the logical topology design problem without wavelength conversion in the network is proposed. We compare our new proposed TS algorithm with previous SA [1] approaches. Our motivation is to compare different meta-heuristics to find a feasible algorithm of logical topology design for network operators to optimize the network. The rest parts of this paper are organized as follows: Section II describes the logical topology design problem; Section III introduces simulated annealing (SA) algorithm; Section IV describes the tabu search (TS) method; Section V shows the simulation model and numerical result; Section VI presents numerical results; Section VII concludes this paper.

\section{II.LOGICAL TOPOLOGIC DESIGN PROBLEM}

\section{A.Network Model}

Consider a $N$-node network with an arbitrary but connected physical topology, in most of the cases there are main four limitations to design the logical topology: 1) The number of tunable transmitters and receivers at each node is limited, that is to say the degree of the node is limited, $P$ (where $P<N$ ) ports at each node. Each node can originate or terminate at most $P$ lightpaths; 2) The number of wavelength on each fibre is also limited, say, $W$ wavelengths on each fibre; 3 ) The wavelength can not be used by different lightpaths on the same fibre; 4) Without the wavelength converter the lightpath has to use the same wavelength along the path. The last two limitations are constraints on the routing and wavelength assignment problem.

\section{B.Objectives}

Given the physical network topology and traffic flows information between end nodes, optimal logical topology design yields a better topology from the viewpoint of IP layer that reduces the traffic load on the electronic routers. Most of 
studies attempt to create a logical topology with minimum network congestion $\lambda_{\max }$. This paper also focuses on the network congestion optimization.

$$
\lambda_{\max }=\max _{i j} \sum_{s d} \lambda_{i j}^{(s d)}, \quad \forall(i, j)
$$

Here, notation $\lambda_{i j}^{(s d)}$ refers to the traffic on the lightpath originating from node $i$ and terminating at node $j$, where the traffic flow starts from the source node $S$ to the destination node D. $\lambda_{\max }$ refers to the load on the most congested logical link.

\section{Logical Topologic Design Subproblems}

The full logical topology design problem can be approximately decomposed into four subproblems: 1) Topology design subproblem is to design a logical topology; 2) Lightpath routing subproblem means to find a route from source to destination in physical networks for each lightpath corresponding to each logical link; 3) Wavelength assignment subproblem is to assign wavelength to each lightpath along the route found in subproblem $2 ; 4$ ) Traffic routing subproblem is to route IP traffic on the logical topology.

Normally, the logical topology design problem is usually formulated as a Mixed Integer Linear Programming [2]-[4], but an exact formulation of this problem quickly grows intractable with increasing size of the networks. In fact, this problem and some of its subproblems are known to be $N P$-hard [3], [4], and [5]. Thus for large size networks, it is not practical to attempt to solve this problem exactly. So the feasible heuristic is very important especially for large size networks. There are various studies proposing heuristic methods for arbitrary logical topologies design [5], [7]. And simulated annealing method, as meta-heuristic methods was also proposed to design logical topology in IP over WDM networks [3]. The previous studies focus on using SA to find the solution combined with a new indirect neighbourhood discovery to find the near optimal solution [1], [6]. It is based on a partial exploration of the space of admissible solutions using swap node pair list approach, finalized to a good solution. For the new swap node pair list approach proposed, our previous studies are focusing on the possible schemes to swap the node pairs in the node pair list. Both of wide indirect neighbourhood discovery (WIND) and Xswap WIND methods had been proposed [6]. But for there is no search memory in the SA algorithm, so it can not be avoided to revisit the space which has been explored in previous searches, to address this problem, this research uses the tabu search approach to record the solutions which have been explored in before to avoid cycling search.

\section{Simulated AnNeAling}

The SA is one of the best-known meta-heuristics. It is based on a partial exploration of the space of admissible solutions, finalized to a good solution. Several SA methods were proposed for designing logical topology in IP over WDM optical networks in the previous studies [1], [6]. In [1], the wide indirect neighbour discovery (WIND) and Xswap WIND methods have been proposed, due to the space limitation, for further details, the reader is referred to [1], [6] and the references therein.

\section{A.Node Pair List and Logical Topology}

For a given physical network topology $G_{p}=(V, E), V$ is the complete set of the nodes in $G_{P}, E$ is the edge set. The logical topology design here is to create lightpath between the source-destination node pair to form the logical topology under a given constrains. Given a source-destination node pair list $Q$, there are totally $M$ elements in the list for all possible node pairs, $\left(S_{i}, D_{i}\right)$ is a source-destination node pair, $i(1 \leq i \leq M)$ is the index of the element in the list $Q$. A node pair list sample is shown in Figure 1.

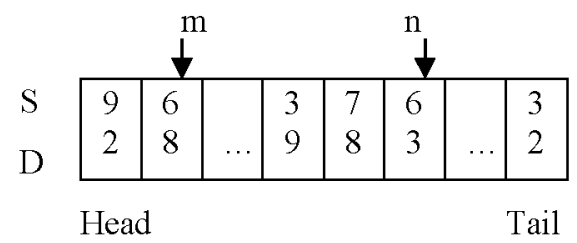

Fig.1 Source-destination node pair list.

For a given node pair list $Q$, to construct a logical topology, lightpaths are created one by one according to the order of the node pair list under the logical degree and wavelength constrains. By preparing the node pair list in certain order related to traffic conditions, traffic conditions can be taken into account to generate a new logical topology. In this indirect neighbour discovery method, a node pair list represents a logical topology; to change the order of the node pair list is possible to generate a new logical topology. The key point in the indirect neighbour discovery approach is the way to change the order of the node pair element in list $Q$. Some of the schemes have been proposed to change the order of the node pair elements in list $Q$. Node pairs can be randomly selected to swap within whole node pair list, this is called wide indirect neighbour discovery (WIND). As for big change or small change, to do multiple node pair swap operations in one step is call Xswap WIND, the Xswap has been found better than WIND in the design [1].

\section{B.Initial Solutions}

In general, the SA search starts from a randomly generated logical topology. An efficient initial solution has been proposed in the previous study [6]. For an efficient initial logical topology, the heuristic logical topology design algorithm (HLDA) is modified, which prepares a source-destination node pair list $Q$, and arranges the list $Q$ in descending order of a certain metric $q_{i j}$ at the first step, then creates the logical links. The sorting metric of the source-destination node pair list is shown as follows:

$$
\begin{gathered}
q_{i j}=\lambda^{(i j)} \times h^{i j} \\
q_{i j}=\lambda^{(i j)}
\end{gathered}
$$




$$
q_{i j}=h^{i j}
$$

where $\lambda^{(i j)}$ and $h^{i j}$ denote the traffic amount and the number of physical hops from source node $i$ to destination node $j$. Here for each design, the best heuristic among these three algorithms is chosen as the initial solution.

\section{Logical Topology Construction Procedures and IP Traffic Routing}

The logical topology construction including wavelength routing and the assignment algorithm are given below:

Step 1: Select the source destination pair $\left(i_{\max }, j_{\max }\right)$ that $q_{i \max , j \max }$ $=\max _{i, j}\left(q_{i j}\right)$; if all source-destination pairs with non-zero traffic have been tried, then go to step3;

Step2: If node $i_{\max }$ and $j_{\max }$ are of fewer degree than $P$ (degree limitation at each node) then

Find lowest available wavelength on the pre-computed minimum physical hop path between $i_{\max }$ and $j_{\max }$ in physical topology. (If there is more than one shortest path, scan them sequentially search the least load path)

If wavelength is available then

Create lightpath and the logical link between node $i_{\max }$ and $j_{\max }$ in logical topology $G_{i}$;

Else

$$
g_{\text {imax } j \text { max }}=1 ; q_{\text {imax }, j \text { max }}=0 ; \text { go to step } 1 ;
$$

$$
g_{i \max j \max }=0 ; q_{i \max , j \max }=0 ; \text { go to step } 1 ;
$$

Else

$$
g_{\text {imax } j \max }=0 ; q_{\text {imax }, j \max }=0 ; \text { go to step } 1 ;
$$

Step3: If we do not yet have $P N$ logical link, place as many remaining logical links as possible randomly so that degree constraints are not violated and a wavelength can be found on the shortest path for the logical link. Otherwise end the algorithm.

Regarding the IP traffic routing subproblem, for simplicity, after creating logical topology, shortest-path-first (SPF) routing is used to route IP traffic on the logical topology. Then, the network maximum congestion in the logical topology is calculated.

\section{TABU SEARCH}

\section{A.Tabu Search Introduction}

The approach presented here uses the tabu search (TS) meta-heuristic. Tabu search is one of the well-known meta-heuristics used to solve the hard combinatorial optimization problems which was first proposed by Glover [8]. It is based on a partial exploration of the space of admissible solutions, finalized to a good solution. For a given problem $A$, and the objective function $f(x), x_{o}$ is the initial solution, $S$ is the solution space, $x$ is a solution in the solution space ( $x \in S$ ), the objective of the tabu search is to search the solution space $S$ from the initial solution $x_{o}$ to try to find the optimal or near-optimal solution $x_{F}$. For any given solution $x(x \in S)$, neighbourhood $\pi(x)$ is a set of solutions obtained by a specified operation on $x$, the operation is called a move, the solution $x(x \in S)$ used to generate the neighbourhood $\pi(x)$ is call current solution. Each element in the neighbourhood is the candidate for the next current solution. During the search, an FIFO limited list is built to keep track of the solutions which had been searched in before, this list is called Tabu list, the length of the list is the size of the tabu list, the tabu list is used to avoid cycling search [9], [10], and [11].

This research employs the indirect neighbourhood discovery method to generate the neighbourhood, the move to generate neighbourhood is defined as the Xswap WIND. The tabu list stores the summary information of solutions which have been searched in before

\section{B.Tabu Search Algorithm}

The TS algorithm involves the following steps:

Step1: Initialize the tabu search list, the best move, and other relative parameters;

Step2: Select an initial solution on the physical topology. The modified heuristic logical topology design algorithm (HLDA) is employed to get the initial solution [1];

Step3: Then implement the Xswap WIND in [1] to generate a new neighbourhood on the current solution, which is the set of the new solutions. Ensure that the solutions are not in the tabu list, and then update the tabu list;

Step4: Calculate fitness value for the individuals in the new neighbourhood, and select the best solution in the neighbourhood as the next current solution. If the selected solution is better than the best solution until now, then update the best solution;

Step5: If the stop condition is not meet, then go back to Step2, otherwise end the search.

\section{The Tabu List}

This research employs Xswap WIND neighbourhood discovery to generate the neighbourhood for tabu search algorithm. In Xswap WIND approach, rather than to change one node pair only, this approach tries to change a group of node pair in the list in one operation, $X$ means the operation contains $X$ WIND swaps. The problem in the Xswap WIND is that it can not avoid revisiting the space which has been explored before, for there is no search memory to record which directions have been tried before. The tabu list tries to record some clues of the solutions which have been explored in the previous explorations.

The tabu list is a fixed size FIFO list TB, the list is shown in Figure 2, the length of the list is $L, T_{i}(1 \leq i \leq L)$ is an element in TB. Each element represents a clue that some pattern of the solutions which have been searched in before. For the list is an FIFO list, so after a pattern of solution has been search in one time, and then it will not be revisited for the length of the tabu list.

The element $T_{i}$ in the tabu list TB can be defined in different approaches. The simplest way is the node-based tabu (NBT), the nodes which have been selected during the operation are inserted into the TB as a $T_{i}$. Another way is to use the node pair $\left(S_{i}, D_{i}\right)$ as the tabu element in the tabu list, the node pairs which have been selected is inserted into the tabu list, this approach is 
called node pair based tabu (NPBT). Both of NBT and NPBT tabu are focusing on the nodes in the physical topology, the swap operation itself can be used as the tabu information. In WIND or Xswap WIND, the basic operation is to select two indices in the node pair list $Q$ and swap the two node pairs in the selected index, the indices which have been used in the operation will be inserted into

\begin{tabular}{|l|l|l|l|l|l|l|}
\hline$T_{1}$ & $T_{2}$ & $\ldots$ & $T_{i}$ & $T_{i+1}$ & $\ldots$ & $T_{L}$ \\
\hline
\end{tabular}

Fig. 2 Tabu list.

the tabu list as one tabu element, this approach is called node pair index based tabu(NIBT). As for the Xswap WIND, the operation itself is uncertain, one operation consists of X-step WIND operations, so NBT, NPBT and NIBT can not track the searched information well for the Xswap. A better way is to treat the whole node pair list as a tabu; it is called node pair list based tabu (NLBT).

\section{The Proposed Tabu List Scheme}

In the NLBT, the node pair list is treated as the tabu information. Considering one $\mathrm{N}$-node network, the degree constrain is $P$, if the wavelength is not considered, there are $(P \times N) / 2$ lightpaths can be created over the physical network. In the indirect neighbourhood discovery approach, the size of the node pair list is $(P \times N) / 2$. When the size of the network increases, the size of the node pair list is rather big. If the whole node pair list is used as the tabu information, then the content of the tabu list should be very larger, and the tabu verification process has great impact on the performance of the whole search, besides this, the tabu search does not try to remember one searched solution only but to remember a direction which have been searched in before. So in this study, the summary information of the node pair list is treated as the tabu information for the Xswap WIND based logical topology design.

The previous studies show that the positions of node pairs in both head and tail of the list are not necessary to be changed, for the lightpaths are created one by one according to the order of the node pair element in the list, the node pairs in the head of the list have more priority to be allocated resources, but the node pairs in the tail in most of the time can not be allocated with resources. This means that the middle part of the list has more contribution to the network congestion optimization. The way of creating lightpaths with limited resource for node pairs in the middle of list affects the final network performance of logical topology, so it is better to limit the search space in the middle of the list. According to this idea, the design of the element in the tabu list tries to record some summary information of solutions which has the close pattern in the middle of the list. This research tries to find a scheme to store the node pair list patterns which have been visited in the previous searches.

For a solution $x$, the following information are treated as the tabu information of the solution.

$$
T_{x}=\sum_{k=m}^{n} k \times\left(S_{k}^{x}+D_{k}^{x}\right), 1 \leq m<n \leq M
$$

where $S_{k}^{x}$ and $D_{k}^{x}$ denote the $k$ th source destination node pair in the node pair list $Q$ of solution $x$. The information of the element in the tabu list is just the summary information of a specific sorted node pair list which represents a solution. In this way, the algorithm tries to record the solutions which have been visited. This algorithm employs only a part of the node pair list as the tabu information, even for the selected part, the list has been summarized. So the possibility to find a new and better solution is increased. The parameters $m$ and $n$ should be adjusted to the most sensitive part of the list, according to the previous discussion, it is better to put them in the middle belt of the node pair list.

\section{NUMERICAL RESULTS}

In this section, a case study on 11-node network topology as shown in Figure 3, is presented. We give the numerical results using randomly generated traffic patterns (but not the actual or absolute value of the traffic) with uniform distribution.

The parameters of the SA are set: the major parameters are initial temperature $T_{0}=200$, cooling parameter $\alpha=0.996$, maximum acceptance counter $P_{a}=10$, maximum iteration counter $P_{t}=100$ at each temperature (for the definition of each parameter, please see [1]). The size of the neighbourhood is 200 for tabu search; the tabu list length is 50 , the $m, n$ are tuned to 20, 40 separately. Each computation is 5000 iterations and repeated 30 times. The solutions are collected and the results are shown in Table 1. Each computation is performed on an Intel Pentium $42.8 \mathrm{GHz}$ Linux-based PC, the memory is 512 $\mathrm{MB}$.

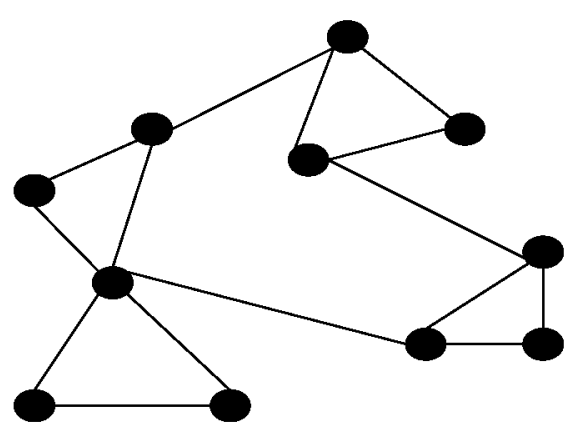

Fig.3 11-Node network topology.

TABLE 1 CONGESTION COMPARISONS FOR 11-NODE NETWORK

\begin{tabular}{c|c|c|c|c|c}
\hline Approaches & $P$ & Average & Max & Min & Time(s) \\
\hline WIND SA & 4 & 1871.2 & 2061 & 1815 & 95 \\
\hline Xswap SA & 4 & 1846.0 & 2031 & 1799 & 92 \\
\hline TS & 4 & 1822.2 & 1861 & 1780 & 107 \\
\hline WIND SA & 6 & 1322.0 & 1389 & 1104 & 111 \\
\hline Xswap SA & 6 & 1253 & 1357 & 1139 & 113 \\
\hline TS & 6 & 1226.2 & 1364 & 1129 & 126 \\
\hline WIND SA & 8 & 1019.6 & 1037 & 975 & 125 \\
\hline
\end{tabular}




\begin{tabular}{c|c|c|c|c|c}
\hline Xswap SA & 8 & 989.6 & 1020 & 779 & 124 \\
\hline TS & 8 & 964.9 & 1027 & 773 & 138 \\
\hline
\end{tabular}

Table 1 shows the results of 11-node network case. The solutions found by different approaches, WIND SA, Xswap SA and TS, respectively are compared. The logical degree is set to $\mathrm{P}$ in $\{4,6,8\}$ and $\mathrm{W}=100$. The results show that among these three approaches, TS outperforms other two methods on the average performance denoted by "Average", the best solution denoted by "Min" and the worst solution denoted by "Max" in the table, the average computation time denoted by "Time".

\section{DISCUSSION}

There is no search memory in the SA algorithm, so it can not be avoided to revisit the space which has been explored in previous searches, to address this problem the tabu search approach records the solutions which have been explored before to avoid cycling search. Figures 4, 5, and 6 show the change of congestion in an average computation cycle. In Figures 4, 5, and 6, tabu search method has a good result at the end of the computation, the reason is that the tabu search method has the search memory to remember the explore space, so compare with other methods, it can enlarge the search space, and get a good result finally.

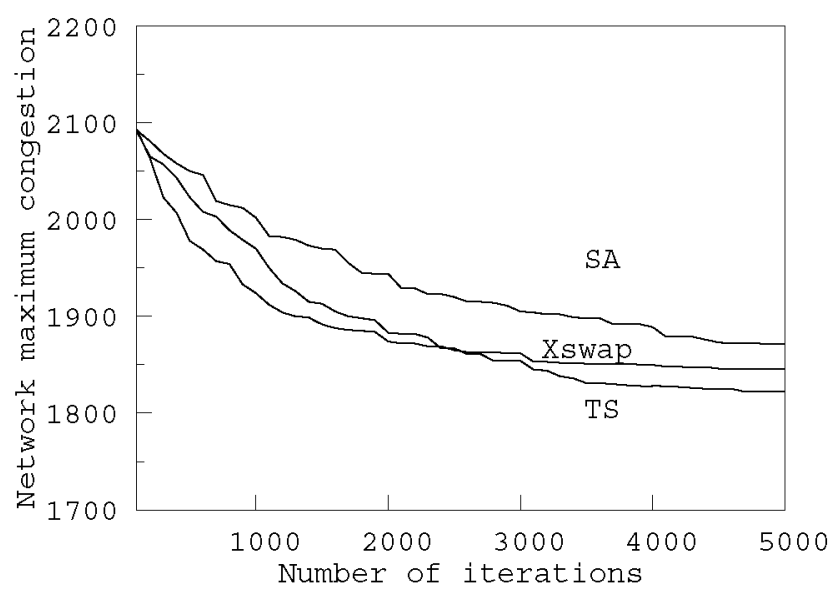

Fig.4 Congestion comparison for degree $P=4$.

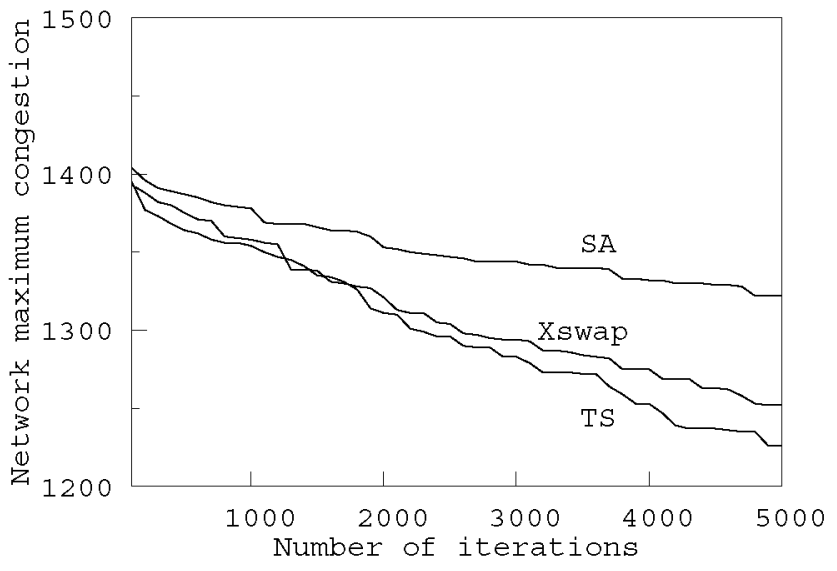

Fig. 5 Congestion comparison for degree $P=6$.

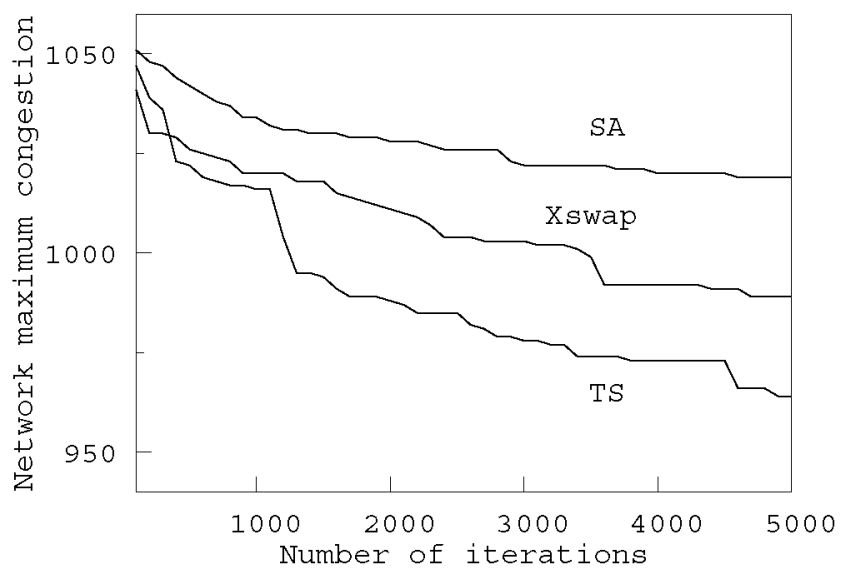

Fig.6 Congestion comparison for degree $P=8$.

\section{CONClusions}

A meta-heuristic tabu search method is proposed to design logical topology in IP over WDM networks. The tabu search algorithm uses the memory mechanism to record some clues for the solution search. The results show that the proposed tabu search method has better performance than the conventional methods for the IP over WDM network logical topology design problem.

\section{REFERENCES}

[1] S. Xu, N. Koyama, and Y. Tanaka, "A fast logical topology design with simulated annealing in IP over WDM networks," Proc. Int. Conf. Information Technology \& Applications (ICITA2004), Harbin, China, pp.21-26, Jan. 2004.

[2] B. Mukherjee, D. Banerjee, S. Ramamurthy, and A. Mukherjee, "Some principles for designing a wide-area WDM optical networks," IEEE/ACM Trans. Networking, vol.4, no.5, pp.684-696, Oct. 1996

[3] R. Ramaswami, and K. N. Sivarajan, "Design of logical topologies for wavelength-routed optical networks," IEEE J. Select. Areas Commun., vol.14, no.5, pp.840-851, June 1996.

[4] R. M. Krishnaswamy, and K. N. Sivarajan, "Design of logical topologies: a linear formulation for wavelength routed optical networks with no wavelength changes," Proc. IEEE INFOCOM, pp.919-927, Oct. 1998 .

[5] D.Banerjee and B.Mukherjee "A practical approach for routing and wavelength assignment in large wavelength-routed optical networks," IEEE J. Select. Areas Commun., vol.14, no.5, pp.903-908, June 1996

[6] S. Xu, N. Koyama, and Y. Tanaka, "A logical topology design with simulated annealing in wavelength routed IP networks," Proc. Int. Network Optimization Conf. (INOC2003), Evry/Paris, France, pp.587-592, Oct. 2003

[7] R. Dutta and G. N. Rouskas, "A survey of virtual topology design algorithms for wavelength routed optical networks," Optical Networks, vol.1, no.1, pp. 73-89, Jan. 2000

[8] F. Glover and M. Laguna, Tabu Search, Kluwer Academic Publishers, Boston, MA 1997

[9] A. Grosso, E. Leonardi, M. Mellia, and A. Nucci, "A logical topologies design over WDM wavelength routed networks robust to traffic uncertainties," IEEE Communications Letters, vol.5, no.4, April 2001

[10] A. Todimala, and B. Ramamurthy, "Survivable virtual topology routing under shared risk link groups in WDM networks," Proc. First International Conference on Broadband Networks (BROADNETS'04), pp.130-139, Oct. 2004.

[11] D. O. Khyda, S. Chamberland, and S. Pierre, "Improvement of routing and wavelength assignment in WDM networks using tabu search", Electrical and Computer Engineering, vol.2, pp.765-768, May 2003. 